\title{
The anti-tumoral effects of the oxygen carrier YQ23 in a triple-negative breast cancer syngeneic model
}

\author{
Elysian Chow, Jonathan S. H. Lau, Terence Wai, Ian P. Y. Lam \\ New Beta Innovation Limited, 18/F Chevalier Commercial Center, Hong Kong, China \\ Contributions: (I) Conception and Design: E Chow, IPY Lam; (II) Administrative support: JSH Lau, IPY Lam; (III) Provision of study materials: JSH \\ Lau, IPY Lam; (IV) Collection and assembly of data: JSH Lau, E Chow, IPY Lam; (V) Data analysis and interpretation: JSH Lau, E Chow, IPY Lam; \\ (VI) Manuscript writing: All authors; (VII) Final approval of manuscript: All authors. \\ Correspondence to: Ian P. Y. Lam. 18/F Chevalier Commercial Center, Hong Kong, China. Email: py.lam@newbetainnovation.com.
}

\begin{abstract}
Background: Triple-negative breast cancer (TNBC), an aggressive breast cancer subtype, is associated with poor prognosis and high mortality rate. In the search for effective therapeutic options, preclinical studies have suggested using systemic oxygenation to inhibit tumor growth and metastasis in various cancer models, including TNBC, by weakening the hypoxia-A2A adenosine receptors (A2AR)-driven immunosuppression in the tumor microenvironment (TME). In our present study, a hemoglobin-based oxygen carrier (HBOC) "YQ23" was tested for its role in modulating the TME and tumor inhibition.

Methods: A syngeneic TNBC mouse model was established by inoculating 4T1 cells subcutaneously in BALB/c mice. Tumor $\left(\sim 100 \mathrm{~mm}^{3}\right)$ bearing mice were treated either with saline or YQ23 (400 mg/kg) i.v. once weekly. To prove the immune-regulatory role of $\mathrm{YQ} 23, \mathrm{CD}^{+}$and $\mathrm{CD}^{+}$cells were depleted from a group of mice prior to treatment. Tumor growth was monitored for four weeks while xenografts were isolated at the end of the treatment for ex vivo immunohistological examination.

Results: YQ23 significantly inhibited the tumor growth, and this suppressive effect was abolished by depleting the host immune cells. Immunohistochemical staining of xenograft sections showed YQ23 reduced the level of hypoxia and adenosine producing ecto-enzyme CD73. Although there was no significant difference in the make up of the intra-tumoral immune populations, we observed a down-regulation of the immune checkpoint PD-1. In concordance with the weakened immunosuppression, the inflammatory cytokine interferon $\gamma$ and cytolytic granzyme B were upregulated.
\end{abstract}

Conclusions: YQ23 treatment may be a potential therapeutic strategy to modulate the TME in TNBC.

Keywords: Adenosine; hypoxia; triple-negative breast cancer (TNBC); xenograft

Submitted Aug 21, 2020. Accepted for publication Dec 12, 2020.

doi: $10.21037 /$ tcr-20-2768

View this article at: http://dx.doi.org/10.21037/tcr-20-2768

\section{Introduction}

Hypoxia in solid tumors results in the development of an environment favoring the up-regulation of genes predisposing cancer cells to a more malignant phenotype. Due to this, hypoxia indicates a reduced overall survival for cancer patients (1-3). The tumor microenvironment (TME) encompasses a wide variety of cellular and non-cellular components, and plays a critical role in the development of cancer, disease progression, and disease outcome through the modulation of stimulator and inhibitory elements. In the TME, hypoxic environments result from a combination of the high metabolic rate of cancer cells, aberrant angiogenesis, and leaky vascularization (4). The hypoxic environment also renders cancerous cells resistant to chemotherapy, radiotherapy, and immunotherapy $(5,6)$.

A central adaptive response of tumor cells to a hypoxic environment is the activation of the heterodimeric oxygensensitive transcriptional activator hypoxia-inducible factor $1-\alpha$ (HIF-1 $\alpha)$. HIF- $1 \alpha$ regulates the expression of over 
100-downstream genes to control vital biological processes required for tumor survival and progression. As a promoter of tumor growth, HIF-1 $\alpha$ is engaged in the modification of gene expression intrinsic in rapidly growing tumors and a driver of glycolysis through multiple targets in the glycolytic pathway in an effort to meet cellular energy demands through the Warburg effect $(7,8)$. In addition to metabolic effects in tumors, HIF-1 $\alpha$ expression has been linked to the development of chemo-resistance in breast cancer stem cells (9). Additionally, HIF-1 $\alpha$ promotes tumor invasiveness and metastasis through the expression of angiogenic and permeability factors, such as vascular endothelial growth factor (VEGF) and matrix metalloproteinases (MMP). Both genes are involved in the remodeling of the extracellular matrix and promote cancer cells intravasation into the blood stream, and thus metastasis (10-13).

Tumor hypoxia and the activation of HIF-1 $\alpha$ are also critical to immune evasion mechanisms employed by cancer cells, which results from modulating the TME into a local immunosuppressive environment. Hypoxia within solid tumors have been shown to trigger increases in chemoattractants for the formation, recruitment, and infiltration of a large number of immunosuppressive cells, such as T-regulatory (Treg) cells and tumor-associated macrophages (TAM) (14-17). Hypoxic TMEs also result in reduced $\mathrm{T}$ cell infiltration, thereby limiting the effects of immune checkpoint blockade therapy, such as PD-1 treatment (18-20).

In addition to these effects, HIF-1 $\alpha$ has also been shown to be capable of modulating the PD-L1 signaling pathway. Prior reports have indicated that under hypoxic conditions, HIF-1 $\alpha$ upregulates PD-L1, leading to immune evasion $(9,17,21)$. HIF-1 $\alpha$ is also responsible for the upregulation of CD73 cell surface expression, which results in the production of extracellular adenosine in the TME (9). Adenosine has been shown to induce a blockade of $\mathrm{T}$ cell and $\mathrm{NK}$ cell function by binding to the A2A adenosine receptor (A2AR) $(22,23)$. The reduced activity of immune cell strongly favors tumor growth. Considering the effects of hypoxia and HIF-1 $\alpha$ on cancer progression, anti-hypoxia treatments and therapies have garnered great interest. Many treatments and proposals have been aimed at reversing the low-oxygen levels of solid tumors to re-sensitize these hypoxic tumors to established therapeutic treatments.

Hyperbaric oxygen therapy (HBOT) has been tested in multiple in vivo models. In triple-negative breast cancer (TNBC), breast cancer, and ovarian cancer, HBOT alone and in combination with other treatments significantly reduced tumor growth (24-26). The use of hemoglobinbased oxygen carriers (HBOCs) are one group of treatments with some demonstrated efficacy at raising the intra-tumoral oxygen level. The resulting intra-tumoral oxygen level from HBOC treatment led to the sensitization of tumors towards chemotherapy in a number of models, which includes hepatocellular carcinoma, TNBC, and esophageal squamous cell carcinoma (26-28). These studies have strengthened the case for using HBOCs for therapy, and have provided evidence supporting the use of HBOCs in other cancer types where hypoxia presents a hindrance to effective therapies.

A few publications have described the efficacy of the oxygen carrier YQ23, a stabilized non-polymeric crosslinked tetrameric hemoglobin, as a novel chemo-sensitizing agent against liver and esophageal cancers (27-29). In vivo YQ23 treatment has also been shown to reduce HIF$1 \alpha$ expression, indicating that YQ23 has the capability to target and reverse the hypoxic nature of solid tumors (27). Here we look to further characterize the effects of YQ23 as an anti-hypoxia drug by using an in vivo TNBC immunocompetent mouse model by using the highly metastatic 4T1 cell line $(30,31)$. Our aim was to determine whether increased oxygenation of a subcutaneous tumor would improve the response of the host immune system.

By utilizing an immune-competent syngeneic animal model, we were able to evaluate the anti-hypoxic effects of YQ23, and the related downstream effects of YQ23 on antitumor immune responses. Our findings suggest YQ23 to be an effective therapy for solid tumors and warrant further investigation into the efficacy of YQ23 in other hypoxic tumors. We present the following article in accordance with the ARRIVE reporting checklist (available at http://dx.doi. org/10.21037/tcr-20-2768).

\section{Methods}

\section{Cell culture}

The murine TNBC cell line 4T1 was a gift from Prof. MS Wong (Hong Kong Polytechnic University). The cells were cultured in RPMI-1640 (Thermo Fisher Scientific, Waltham, MA, USA) medium supplemented with $10 \%$ fetal bovine serum (Thermo Fisher) in a humidified atmosphere with $5 \% \mathrm{CO}_{2}$ at $37^{\circ} \mathrm{C}$.

\section{YQ23 preparation}

YQ23 is a bovine-derived stabilized non-polymeric cross- 
linked tetrameric hemoglobin construct $(65 \mathrm{kDA})$ with undetectable/low levels of dimeric hemoglobin $(32 \mathrm{kDa})$, phospholipid, DNA impurities and protein impurities. The concentration of the YQ23 product is $10 \mathrm{~g} / \mathrm{dL}$, with a $\mathrm{pH}$ range of 7.4-8.4. The osmolality and viscosity at $37^{\circ} \mathrm{C}$ are $250-340 \mathrm{mOsm} / \mathrm{kg}$ and $>0.9$ centipose, respectively. The information for the YQ product is shown in US patent nos. US7,932,356 B1, US 8,048,856 B1 and PCT/US12/46130.

\section{Animal model}

Five to 7 week old, female BALB/c mice (20 to $25 \mathrm{~g}$ in body weight) were purchased from Vital River Laboratory Animal Technology Co. The mice, with free access to food and water, were maintained under specific pathogenfree conditions at the Hong Kong Polytechnic University Shenzhen Research Institute, according to institutional guidelines, with six mice per cage.

All animal studies were performed under a project license (No. 150705) granted by the Laboratory Animal Ethics Committee of the Hong Kong Polytechnic University Shenzhen Research Institute, in compliance with the Guangdong Province Experimental Management Regulations for the care and use of animals. The mice were housed for one week prior to subcutaneous inoculation with $5 \times 10^{5} 4 \mathrm{~T} 1$ cells. When the tumor reached $100 \mathrm{~mm}^{3}$ in volume, mice were randomized into groups for treatment.

\section{YQ23 in vivo study}

The mice were randomized into two groups $(n=6)$, with an average tumor volume of $100 \mathrm{~mm}^{3}$. Six mice were allocated to the control, and six mice were allocated to the YQ23 treatment group as prior experience with the model has shown that this is the minimum number of mice required to produce statistically reliable data. Tumor volume was calculated by using Eq. [1]. One prior murine cancer model used an intravenous dose of $200 \mathrm{mg} / \mathrm{kg}$ YQ23, and two prior YQ23 in vivo rat studies used an intravenous dose of $200 \mathrm{mg} / \mathrm{kg}$, which has a murine equivalent dose of approximately $400 \mathrm{mg} / \mathrm{kg}$ (28-30,32). The mice were administered $400 \mathrm{mg} / \mathrm{kg}$ YQ23 in $100 \mu \mathrm{L}$ of buffer intravenously, or an equivalent volume of saline, once weekly. Tumor volume was measured three times per week. The mice were sacrificed at the end of 4 weeks. The tumor and lung were sampled for further analysis via immunohistochemistry (IHC) and flow cytometry. This experiment was repeated four times.

\section{Immune depletion model}

The mice were randomized into the following two groups of six mice with an average tumor volume of $100 \mathrm{~mm}^{3}$ : immune depletion + saline, and immune depletion + YQ23. All mice were administered $250 \mu \mathrm{g}$ each of anti-CD8 and anti-CD4 antibodies via intraperitoneal injection once weekly (BioXCell, Lebanon, NH, USA). For mice allocated to the immune depletion + YQ23 group, YQ23 was administered at a dose of $400 \mathrm{mg} / \mathrm{kg}$ via tail-vein injection once weekly. An equal volume of saline was administered to mice in the control group. Tumor volume was measured three times per week. Tumor volume was measured twice per week. After 4 weeks, the mice were sacrificed.

\section{Tumor volume}

Following measurement with calipers, the modified ellipsoidal formula was used to calculate tumor volume \{Eq. [1]\}. The tumor volume was then used to calculate the relative tumor volume $\{$ RTV, Eq. [2]\}. The RTV values were then used to calculate the tumor growth inhibition T/ $\mathrm{C}$ ratio $\{\mathrm{T} / \mathrm{C} \%, \mathrm{Eq} .[3]\}$.

Tumor volume $=1 / 2\left(\right.$ length $\times$ width $\left.^{2}\right)$

$$
\mathrm{RTV}=\mathrm{TV}_{\mathrm{n}} / \mathrm{TV}_{1}
$$

Where $T V_{n}$ is the tumor volume at day $n$ and $T V_{1}$ is the tumor volume on day 1 (date on which YQ23 treatment began).

$\mathrm{T} / \mathrm{C} \%=100 \times($ mean $\mathrm{RTV}$ of the treated group $) /($ mean RTV of control group)

The T/C\% was tracked over the course of the study to determine whether the treatment had any therapeutic effects.

\section{Hypoxyprobe-1 plus}

The Hypoxyprobe-1 Plus Kit was purchased from Hypoxyprobe (Burlington, MA, USA). Twenty-five minutes before sacrifice, the mice were administered with $60 \mathrm{mg} / \mathrm{kg}$ of the Hydroxyprobe-1 solution via intraperitoneal injection. The tissue was fixed in $4 \%$ paraformaldehyde for paraffin embedding. The steps for the deparaffinization, antigen retrieval, and antibody staining were as per 
manufacturer's instructions.

\section{IHC}

IHC was performed on paraffin-embedded 4T1 tumors collected from mice in the YQ23 in vivo study. Four to $5 \mu \mathrm{m}$ paraffin-embedded sections were de-paraffinized with xylene and rehydrated with graded ethanol to water. Antigen retrieval was performed by heating the slides in $10 \mathrm{mM} \mathrm{pH}$ 6 citric buffer for $8 \mathrm{~min}$ in a microwave. After endogenous peroxidase blocking with $3 \%$ hydrogen peroxide in methanol for $10 \mathrm{~min}$, the slides were blocked with $10 \%$ serum from the secondary antibody host for $1 \mathrm{hr}$ in PBS. The sections were the incubated in different primary antibodies overnight: rabbit polyclonal anti-CA9 (NB100-417, Novus Biologics, Littleton, CO, USA), rabbit polyclonal anti-CD31 (ab28364, Abcam, Cambridge, UK), rabbit monoclonal antiCD73 (13160S, Cell Signaling, Danvers, MA, USA), rabbit polyclonal anti-granzyme B (ab4059, Abcam), anti-IFN $\gamma$ (sc9344, Santa Cruz Biotechnology, Dallas, TX, USA), antiPD-1 (ab52587, Abcam). Primary antibodies were detected using the appropriate Vectastain Elite ABC HRP Kit (Vector Labs, Burlingame, CA, USA), and visualized with Liquid $\mathrm{DAB}+$ Substrate Chromogen Sytem (Dako, Carpinteria, CA, USA) before counterstaining with hematoxylin. Images were captured using a Leica DM 4000.

\section{Flow cytometry}

Tumor infiltrating lymphocytes (TILs) were isolated by mincing the tumor mass and digesting with collagenase and hyaluronidase for $1 \mathrm{hr}$ in a $37^{\circ} \mathrm{C}$ incubator. After incubation, TILs were passed through a $40 \mu \mathrm{m}$ cell strainer, and washed with $\mathrm{PBS}+2 \% \mathrm{FBS}+5 \mathrm{mM}$ EDTA (Sigma Alrdige, St. Louis, MO, USA). The cells were spun down prior to resuspension in $100 \mu \mathrm{L}$ of FACS buffer containing Fcblocking antibodies for $10 \mathrm{~min}$ at $4^{\circ} \mathrm{C}$. The cells were then incubated in LIVE/DEAD dye (Thermo Fisher) and stained with the following antibodies for $20 \mathrm{~min}$ at $4{ }^{\circ} \mathrm{C}$ : anti-CD3 Alexa Fluor 488 (557666, BD Bioscience, San Jose, CA, USA), anti-CD4 PE-Cy7 (552775, BD Bioscience), antiCD8 PerCP-Cy5.5 (551162, BD Bioscience). The cells were then washed in FACs buffer and re-suspended in PBS + $2 \%$ FBS. The cells were then analyzed with a BD FACSAria III.

\section{Statistical analysis}

Statistical analysis was performed using GraphPad Prism
6. For comparison between the control and treatment group, statistical testing involved the use of the $t$-test. Data presented in bar charts are mean \pm SEM. A P value of less than 0.05 was considered statistically significant.

\section{Results}

To investigate whether YQ23 exerts an immunotherapeutic effect, a syngeneic murine breast cancer model was established. For this model, $400 \mathrm{mg} / \mathrm{kg}$ YQ23 administered intravenously once weekly resulted in a significant decrease in the tumor growth inhibition (T/C) ratio (Figure $1 A$ ). Our data here shows the results from four separate studies combined into one figure, with 13/24 mice analyzed for the control group, and 13/24 analyzed for the YQ23 treatment group (Figure 1A). Twenty-eight days after the start of therapy, the T/C ratio of the YQ23 treated tumor was $47.6 \% \pm 15.98 \%$ that of control (Figure 1A). After sacrifice, lungs were collected from two studies and analyzed for metastatic nodules. It was observed that the number of metastatic nodules in the lung were much lower in the YQ23 treatment group (8/12 mice) compared to control (10/12 mice), with the control group averaging $15.2 \pm 7.35$ and YQ23 treatment averaging $5.1 \pm 2.93$ nodules (Figure $1 B$ ). Mice were not included in statistical analysis if there was no xenograft uptake, the tumor turned necrotic, the initial tumor size was too large or too small, or animals reached a humane endpoint over the course of the study.

The anti-tumor effects of YQ23 were found to be mediated by CD 4 and CD8 T cells. This was shown via host immune depletion of CD4 and CD8 T cells. AntiCD4 and anti-CD8 antibodies were administered prior to YQ23 treatment, which resulted in the depletion of host CD4 and CD8 T cells. In turn, the lack of T cells abrogated the tumor inhibitory effects of YQ23 (Figure 1C). Over the course of this host immune cell depletion experiment, there was no significant difference in tumor size between control and YQ23 treatment after the loss of CD4 and CD8 immune cells (Figure 1C). For this set of statistical analysis, $6 / 6$ mice were included for the immune depletion + buffer, and 4/6 mice for the immune depletion + YQ23.

Due to YQ23's nature as a cross-linked hemoglobin structure capable of delivering oxygen, we next looked into whether YQ23 treatment increased tumoral oxygenation. The mice were administered $60 \mathrm{mg} / \mathrm{kg}$ of hypoxyprobe- 1 via intraperitoneal injection $25 \mathrm{~min}$ prior to sacrifice. As observed in Figure 2A, YQ23 treatment significantly reduced intra-tumoral hypoxia, which was $0.37 \pm 0.16$-fold 


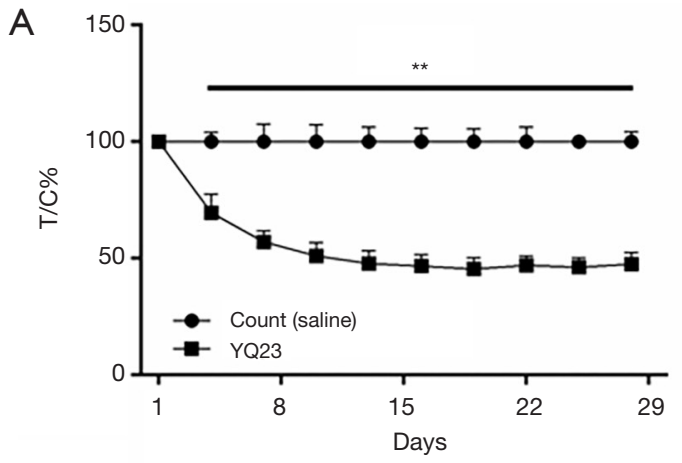

B

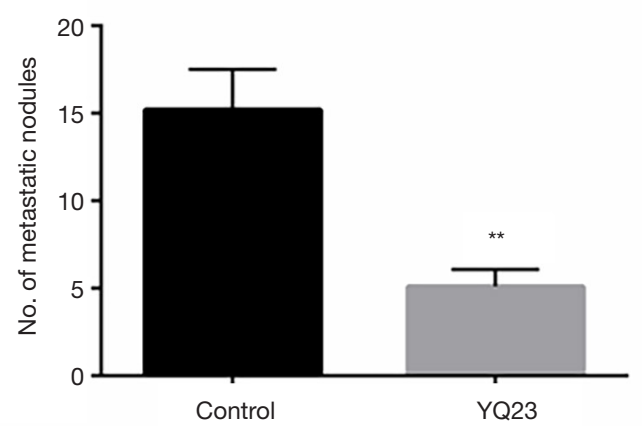

C
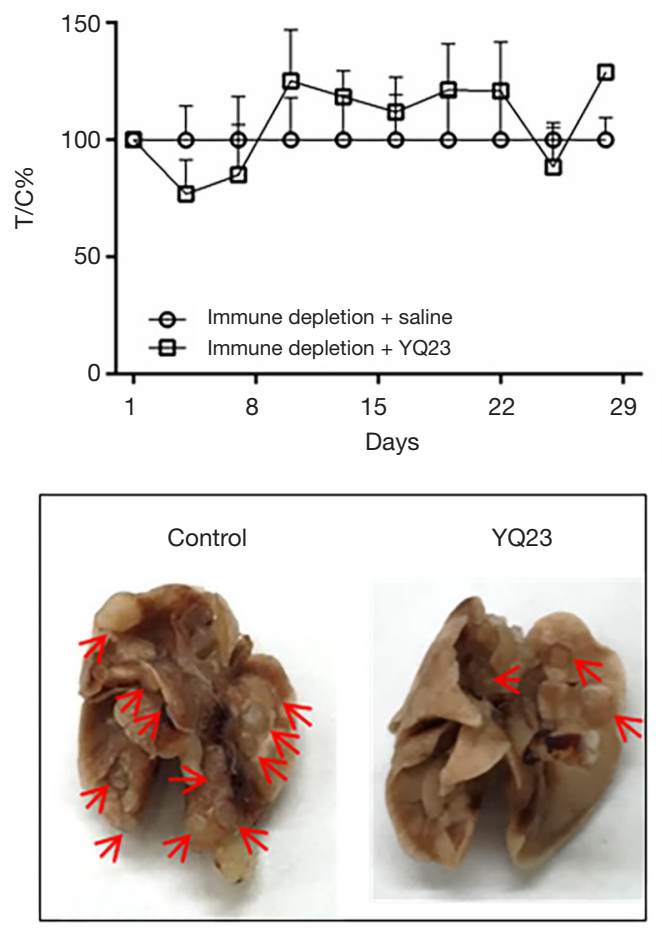

Figure 1 Treatment with YQ23 inhibited the growth of the 4T1 tumor and is mediated by CD4 and CD8 T cell activity. (A) Average tumor growth inhibition ratio (T/C\%) of four independent studies of 4T1 tumors treated with saline or YQ23 over a 28-day period. Thirteen/24 mice were included in the analysis for both the control group and YQ23 treatment group. Unpaired $t$-tests with Welch's correction were used to determine significance between the control group and treatment group on a day to day basis. (B) Treatment with YQ23 reduced the number of metastatic nodules observed in the lung. A representative image for both groups has been provided. The red arrows indicate metastatic nodules. Lung tissue from two studies was analyzed, with 10/12 mice from the control group, 8/12 mice from the YQ23 treatment group. Statistical significance between the control and YQ23 group was determined by using an unpaired $t$-test with Welch's correction. (C) Depletion of host immune cells by anti-CD4 and anti-CD8 antibodies abolished the tumor inhibitory effect of YQ23. Six/six mice were included in the analysis for the immune depletion + saline, and 4/6 mice for the immune depletion + YQ23 group. The error bars are + standard error of measurement. For all figures, ** indicates a $\mathrm{P}$ value of less than 0.01 .

that of the control group. Immunohistological stains were also performed for CA9, a cellular hypoxia biomarker. Here, our analysis of CA9 IHC stains show that YQ23 treatment resulted in a value which was $0.25 \pm 0.07$-fold that of the control staining area (Figure 2B).

As YQ23 treatment resulted in reduced hypoxyprobe-1 and CA9 staining, staining for CD73 expression on 4T1 cells was performed next to determine whether a similar effect occurred. YQ23 treatment significantly reduced CD73 signal intensity by $0.59 \pm 0.14$-fold (Figure $3 A$ ). Flow cytometry was utilized to corroborate this data by counting the number of $\mathrm{CD}^{-} \mathrm{CD}^{-} 3^{+}$cells. The $\mathrm{CD} 3^{-} \mathrm{CD} 73^{+}$subset of cells consists of $4 \mathrm{~T} 1$ cancer cells, as CD3 expression occurs on T cells. The control group had $38.6 \% \pm 12.42 \%$ $\mathrm{CD}^{-} \mathrm{CD}^{-} 3^{+}$cells, whilst YQ23 treatment resulted in

\section{$20.99 \% \pm 8.38 \% \mathrm{CD}^{-} \mathrm{CD}^{-} 3^{+}$cells (Figure $3 B$ ).}

Hypoxia in tumors has been associated with angiogenesis. Due to YQ23 being capable of increasing intra-tumoral oxygenation, we investigated whether there would be a correlating decrease in angiogenesis. In order to determine YQ23's effects upon angiogenesis, tumor sections were stained for CD $31^{+}$(PECAM-1). Images of the stained tumor sections were analyzed to determine the number of intact vessels inside the tumor. Our results indicated that YQ23 treatment resulted in a value of $0.64 \pm 0.08$-fold compared to control (Figure 3C).

PD-1, IFN $\gamma$, and granzyme B staining was also performed, in order to elucidate information about tumoral $\mathrm{T}$ cell activity. Compared to the control group, the YQ23 treatment group had significantly reduced PD-1 
A

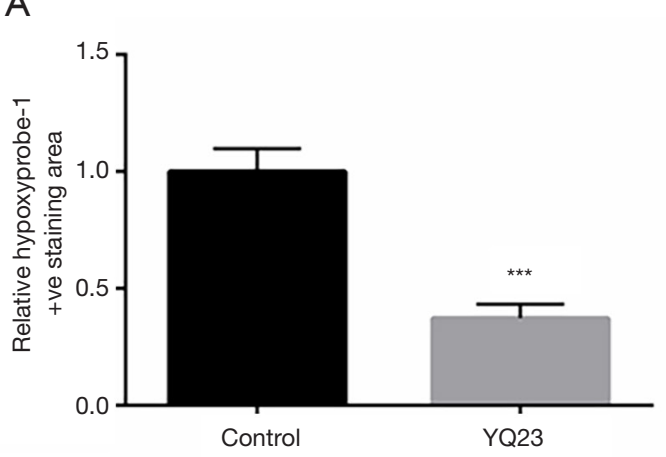

B

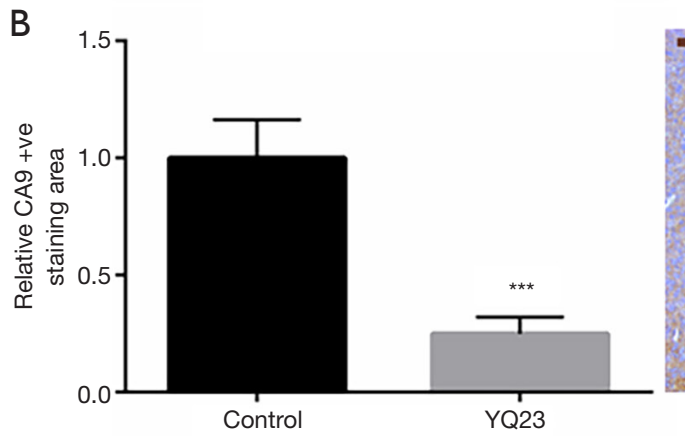

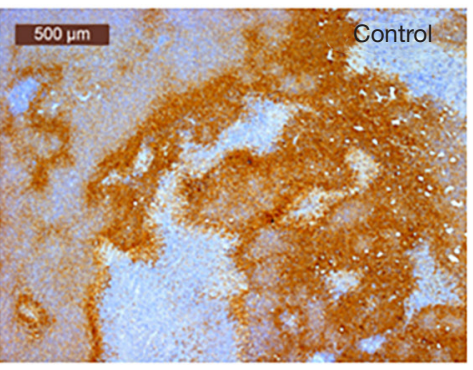
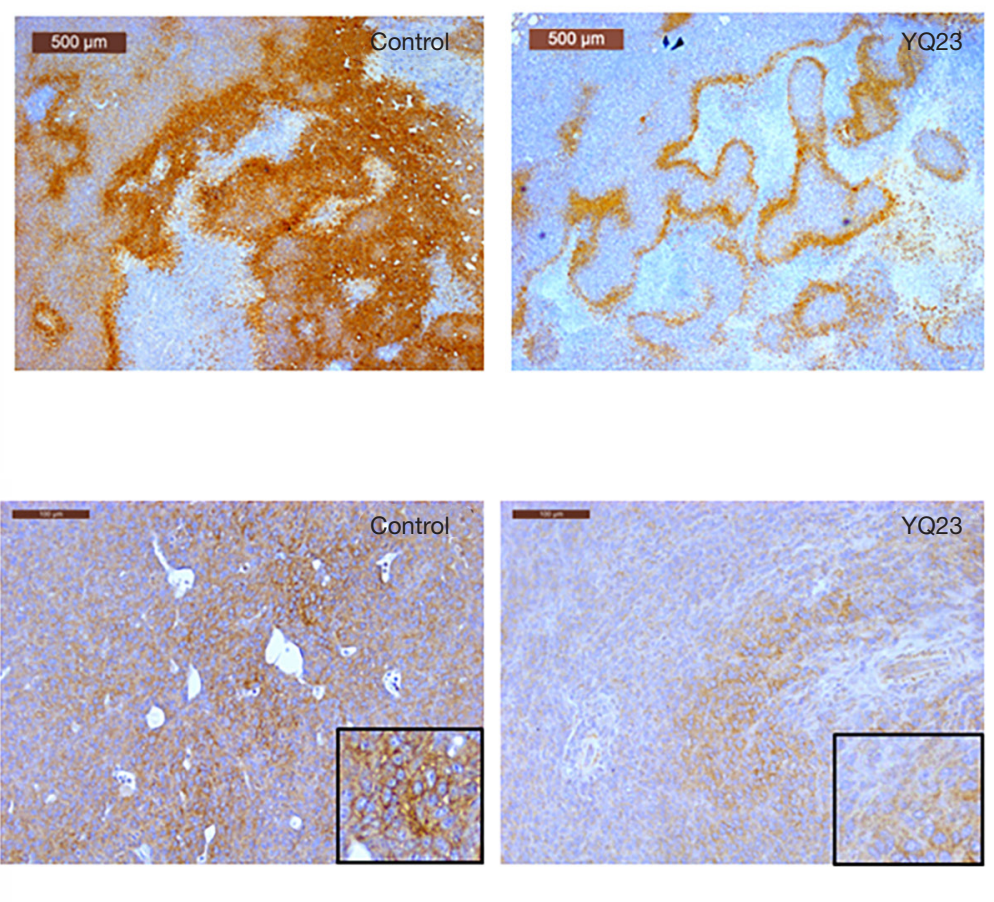

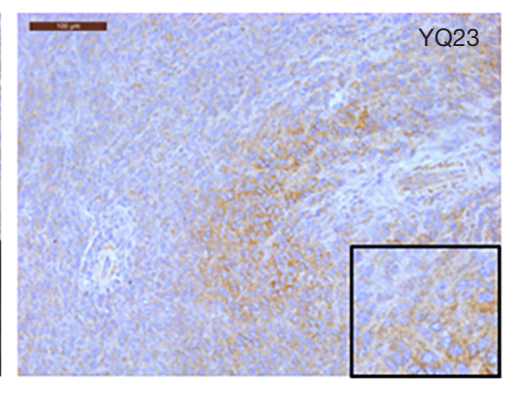

Figure 2 YQ23 treatment reduced the signal intensity of hypoxia markers. (A) Immunohistological staining for hypoxyprobe-1 was performed, with ImageJ used to score stains by positive area. Sections from one study were stained. Representative images of the control ( $5 / 6$ mice) and YQ23 treatment group (6/6 mice) are shown. The scale bar for both images is based on $500 \mu \mathrm{m}$. (B) Immunohistological staining for CA9 was performed, with ImageJ used to score stains by positive area. Three studies were pooled together for analysis. Representative images of the control (11/18 mice) and YQ23 treatment group (8/18 mice) are shown. The scale bar for both images is based on $100 \mu \mathrm{m}$. For all figures, statistical analysis was performed using an unpaired $t$-test with Welch's correction. The error bars are + standard error of measurement. ${ }^{* * *}$ indicates a $\mathrm{P}$ value of less than 0.001 .

expressing cells, with a 0.64 -fold decrease in cells identified as expressing PD-1 (Figure 4A). YQ23 treatment also resulted in a higher number of IFN $\gamma$ expressing cells, with a $1.5 \pm 0.16$-fold increase (Figure $4 B$ ). Flow cytometry analysis of the cells indicated that there was an increase in the number of $\mathrm{CD}^{+} \mathrm{CD}^{+} \mathrm{IFN} \gamma^{+}$cells, but not to a significant degree (Figure $4 C$ ). In contrast to the $\mathrm{CD}^{+} \mathrm{CD}^{+}, \mathrm{YQ} 23$ treatment significantly increased the number of $\mathrm{CD}^{+} \mathrm{CD}^{+}$ IFN $\gamma^{+}$cells, by 2.27 -fold, with $14.81 \% \pm 3.29 \%$ positive cells for control and $33.6 \% \pm 5.46 \%$ positive cells for the YQ23 treatment group (Figure 4D). When tumor sections were stained for granzyme $\mathrm{B}$, there was no significant difference in the number of granzyme B positive cells (Figure 4E).

\section{Discussion}

TNBC is a highly aggressive cancer and metastatic type of breast cancer. Approximately $15-20 \%$ of all breast cancer patients are diagnosed with TNBC (33). The disease primarily presents as invasive ductal carcinomas, and readily metastasizes $(33,34)$. TNBC itself is characterized by the lack of progesterone receptor, estrogen receptor, and human epidermal growth factor receptor 2 (35). Even within TNBC, there are multiple subtypes, ranging from one to six, depending on the system used for characterizing the genomic profile $(36,37)$. TNBC has a high metastatic potential, with metastatic nodules commonly found in the brain, lung, bone, and liver, and hypoxia has been shown to play a role in this (38-40). Clinical performance and performance scores and number of metastatic sites are prognostic factors for disease survival (41). The comparatively low number of potential molecular targets, along with its high metastatic potential makes TNBC treatment difficult (35). Another factor behind the difficulty of treatment for TNBC is that the use of chemotherapy as a line of therapy results in the induction of CD47, CD73, and 

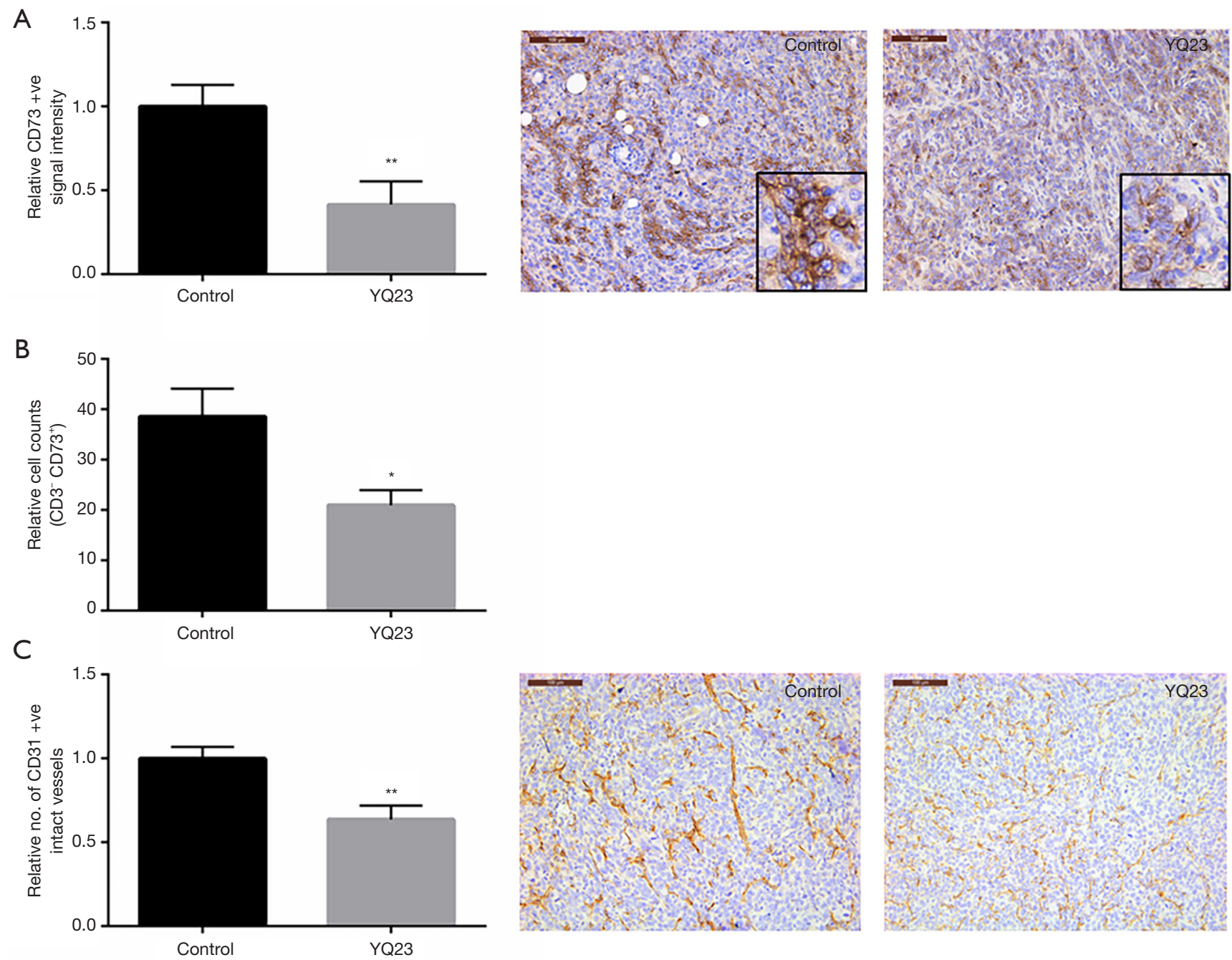

Figure 3 Treatment with YQ23 reduced both CD73 and CD31 expression. (A) An immunohistological stain for CD73 was performed on a tumor section after animal sacrifice. ImageJ was used to calculate the relative signal intensity between the control group and YQ23. Data was pooled together from three studies for analysis. A representative image of the control group (7/18 mice), and a representative image of the treatment group (8/18 mice) is shown. The scale bar for both images is based on $100 \mu \mathrm{m}$. (B) Flow cytometry was used to enumerate CD ${ }^{-}$ $\mathrm{CD} 73^{+}$cells extracted from the tumor. Data from one study was used for analysis, with 5/6 mice for the control group, and 6/6 mice for the YQ23 treatment group. (C) Immunohistological staining for CD31 was performed on a tumor section after animal sacrifice. The stain was used to grade lengths of sections into intact or fragmented vessels, where a CD31 positive stain with a length of three nuclei or more were considered to be intact. Data was pooled across four studies. Representative images of the control group (11/24 mice), and treatment group (11/24 mice) are shown. The scale bar for both images is based on $100 \mu \mathrm{m}$. For all figures, statistical analysis was performed using an unpaired $t$-test with Welch's correction. The error bars are + standard error of measurement. * indicates a $\mathrm{P}$ value of less than 0.05 ; ** indicates a $\mathrm{P}$ value of less than 0.01 .

PD-L1, which has been noted to result from hypoxia $(9,40)$.

Hypoxia in solid tumors has been correlated with poor patient outcomes, which results from downstream effects of the hypoxic TME. The development of a hypoxic TME results in a highly immunosuppressive locale, which reduces the efficacy of both drugs and immunotherapy, yet also results in an area for novel drug development. In the present study, we demonstrate that YQ23 has a strong antitumoral effect in an immunocompetent model. This effect was linked to the host immune system, where host immune 

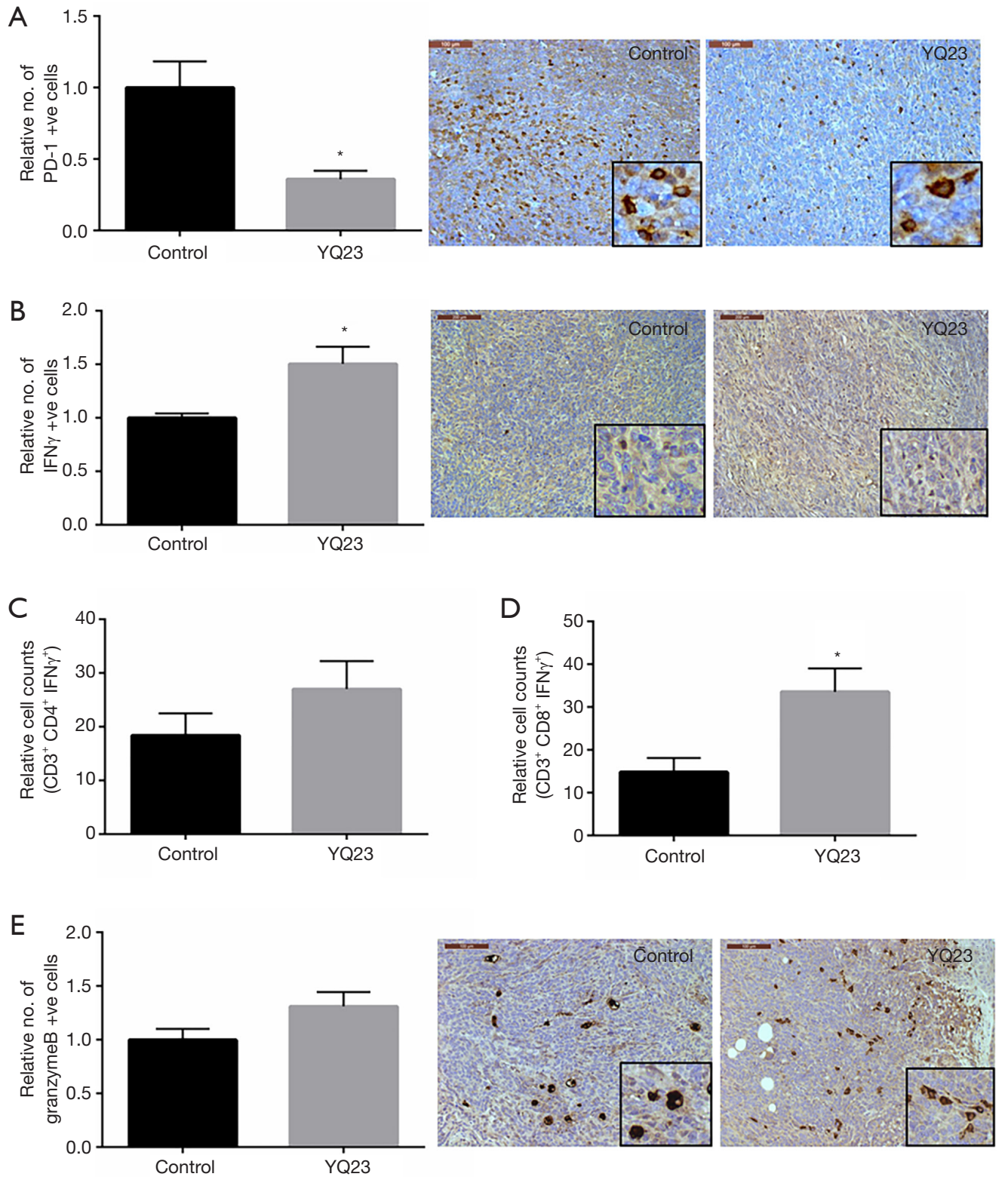

Figure 4 YQ23 treatment enhanced immune cell activity and infiltration. (A) PD-1 positive cells were counted after immunohistochemical staining. Slides from three studies were analyzed, with a representative image for the control group (7/18 mice) and YQ23 treatment group (8/18 mice). The scale bar for both images is based on $100 \mu \mathrm{m}$. (B) IFN $\gamma$ positive cells were counted after immunohistochemical staining. Slides from three studies were analyzed, with a representative image for the control group (6/18 mice) and YQ23 treatment group (8/18 mice). The scale bar for both images is based on $200 \mu \mathrm{m}$. (C) Flow cytometry was performed upon tumor tissue from one study after collagenase and hyaluronidase to identify the number of $\mathrm{CD}^{+} \mathrm{CD}^{+} \mathrm{IFN} \gamma^{+}$cells. The control group included $5 / 6$ mice, whilst the YQ23 treatment group included 6/6 mice. (D) Flow cytometry was performed upon tumor tissue from one study after collagenase and hyaluronidase digestion to identify the number of $\mathrm{CD}^{+} \mathrm{CD}^{+} \mathrm{IFN} \gamma^{+}$cells. Tumor tissue samples were collected from one study, where the control group included 5/6 mice and the treatment group included 6/6 mice. (E) An immunohistochemical stain for granzyme B was performed upon tumor tissue samples across two studies. A representative image for the control group (6/12 mice) and YQ23 treatment group (8/12 mice) has been provided. The scale bar for both images is based on $100 \mu \mathrm{m}$. For all figures, statistical analysis was performed using an unpaired $t$-test with Welch's correction. The error bars are + standard error of measurement. * indicates a $\mathrm{P}$ value of less than 0.05 . 
cell depletion abrogated the anti-tumor effects of YQ23 treatment. We also demonstrated that YQ23 treatment was capable of reducing the number of metastatic nodules in the lung, which corresponds to a prior study investigating liver metastasis (29).

Our investigations into the mechanism of YQ23's effects on the tumor show that the HBOC is capable of reducing hypoxia in the tumor. In lieu of determining HIF- $1 \alpha$ expression, we utilized hypoxyprobe-1, CA9, and CD31 stains in our model. Both CA9 and CD31 have high expression during hypoxia, due to upregulation by HIF-1 $\alpha$ (42-46). CD31, also known as PECAM-1, indicates the presence of endothelial cells (47). Signals for hypoxyprobe-1, CA9, and the number of CD $31^{+}$ blood vessels were reduced in the YQ23 treatment group, indicating reduced hypoxia, as well as reduced metastasis. As HIF- $1 \alpha$ expression is negatively correlated with patient survival, the reduced CA9 and CD31 signals indicate a potentially beneficial effect in a clinical environment.

Further immunohistological staining of tumor sections showed YQ23 treatment reduced CD73 expression on 4T1 cells. CD73 is directly regulated by HIF-1 $\alpha$ and is responsible for immune suppression via generation of extracellular adenosine $(9,22,23,47)$. Elevated expression of CD73 in TNBC has been associated with reduced overall survival in patients, along with chemotherapy resistance, whilst anti-CD73 antibody therapy was shown to significantly reduce tumor volume (48-51). High CD73 expression and the resulting high extracellular adenosine levels have been noted to increase the proportion of regulatory $\mathrm{T}$ cells and the immunosuppressive effects of regulatory $\mathrm{T}$ cells (Tregs) by acting through the A2AR signaling pathway $(52,53)$. Although we did not investigate the number of Tregs here, A2AR signaling pathway inhibition in an in vivo head and neck squamous carcinoma model has been shown to increase the $\mathrm{CD} 8^{+} \mathrm{T}$ cell response, and reduce the number of Tregs (54).

In conjunction with the reduced CD73 expression, we noted a decrease in the number of PD- $1^{+}$cells and a significant increase in IFN $\gamma^{+}$cells. PD-1 expression on T-cells indicates exhaustion and reduced activity (55). As such, the reduced expression of both CD73 and PD-1 after YQ23 treatment potentially indicates increased inflammatory T cell activity as a mechanism of YQ23's actions. For the IFN $\gamma^{+}$cells, a significant increase was observed in the proportion of $\mathrm{CD}^{+} \mathrm{CD}^{+}$cytotoxic $\mathrm{T}$ cells following YQ23 treatment. Although not significant, there also was an increase in the proportion of inflammatory IFN $\gamma^{+} \mathrm{CD}^{+}$Th 1 cells and the number of granzyme B positive cells.

Overall, our results here indicate that increasing tumor oxygenation leads to the TME shifting from an immunosuppressive state to a more inflammatory one in a murine TNBC model. The usage of YQ23 as a therapeutic has potential benefits. One area of interest over the past few years is the use of immunotherapy, which potentiates the effects of the host immune system against targeted cancer cells. However, immunotherapy relies upon immune system activity, which may not be high in an immunosuppressive environment. Although immune cells may be able to identify malignant cancer cells after PD-1, PD-L1, or CTLA-4 antibody therapy, the immune cells may not be active enough to induce apoptosis in malignant cells. As hypoxia attenuates the immune response, there has been a great deal of interest in developing a drug capable of disrupting hypoxia in the TME.

Multiple other methods of addressing intra-tumoral hypoxia by increasing oxygenation have been tested. Although HBOT was previously shown to some benefits to patients undergoing chemotherapy or radiotherapy in a variety of cancer types, there is no conclusive evidence for breast cancer (56). In an in vivo model involving the highly metastatic MDA-MB-231 TNBC in NOD/SCID mice, HBOT reduced metastasis and tumor growth (57). In another set of in vivo breast cancer models, hyperoxia reduced angiogenesis when used in conjunction with 5 -fluorouracil $(58,59)$. However, one of the downfalls to HBOT is the equipment required to treat patients on a wide scale. There also have been issues with HBOT in in vivo models, where rats displayed seizures symptoms (24).

Small molecules, such as efaproxiral have also been tested. Efaproxiral acts by reducing the oxygen binding affinity. It did not pass Phase 3 clinical trials due to a lack of improvement in overall survival for breast cancer patients with brain metastases (60). Trans sodium crocetinate, which acts by allowing faster oxygen diffusion from red blood cells to the vascular wall, is undergoing a Phase 3 trial (61). NVX108 , a fluorochemical, has the ability to increase oxygen dissolution and is being tested as a radiosensitizer (62). YQ23 is different from these small molecules as YQ23 is a tetrameric hemoglobin construct delivering oxygen to hypoxic tissues, rather than changing the physiochemical properties of plasma.

Although there are potential therapeutics affecting A2AR or CD73 activity, these drugs do not cover the full 
range of YQ23's effects and may have off-target effects. AB680, a reversible, competitive CD73 inhibitor, is currently undergoing clinical trials in gastrointestinal malignancies and metastatic castration resistant prostate cancer (63). CPI-444, an A2AR antagonist, was tested in an in vivo colorectal cancer model. Alone, CPI-444 did not result in any change to tumor volume, but when CPI-444 was used in conjunction with anti-PD-1 antibodies, there was an enhanced effect upon survival, and tumor volume was further reduced (64). Unfortunately, one side effect of CPI-444 treatment was increased CD73 expression, which potentially has downstream immunosuppressive effects (64). One benefit YQ23 has over drugs targeting A2AR or CD73 activity is the ability to inhibit both at the same time. During concurrent CD73 antibody blockade and A2AR antagonism, better control of tumor growth and reduced metastasis occurred (65).

Overall, our results have shown that YQ23 alone has a significant effect upon tumor growth. Despite these potential benefits in the clinical setting, there are areas in which further study could be performed. Although we observed the change in expression of several biomarkers, further exploration into other leukocytes in the tumor would expand upon our knowledge of YQ23's effects on the immune system. Another area of interest would be innate immune cells, as hypoxia has also been noted to influence the CD47 don't eat me signal in TNBC. Genomic profiling of the metastatic nodules and xenograft locations could also provide deeper details of the effects of YQ23 treatment and also be used to identify molecular markers which could be used to screen patients. From our study, CD73 stands out as the best marker at this point, as it is upregulated by hypoxia, and plays a key role in immunosuppression.

Future work with YQ23 could investigate into whether a change in survival is observed in an in vivo model. As we have shown that YQ23 reduced CD73 expression, a logical step would be to determine whether the co-treatment of YQ23 along with a PD-(L)1 therapeutic would result in a stronger effect upon tumor growth.

\section{Conclusions}

In summary, YQ23, as a HBOC, can reduce tumor growth by stimulating the host immune response. Mechanistically, this action is performed by reducing tumor hypoxia, which then leads to the downregulation of CD73 and PD-1, followed by increased tumor infiltration and activity by CTLs.

\section{Acknowledgments}

Funding: The work was supported by New Beta Innovation Limited.

\section{Footnote}

Reporting Checklist: The authors have completed the ARRIVE reporting checklist. Available at http://dx.doi. org/10.21037/tcr-20-2768

Data Sharing Statement: Available at http://dx.doi. org/10.21037/tcr-20-2768

Conflicts of Interest: All authors have completed the ICMJE uniform disclosure form (available at http://dx.doi. org/10.21037/tcr-20-2768). All authors report that they are employees of New Beta Innovation Limited.

Ethical Statement: The authors are accountable for all aspects of the work in ensuring that questions related to the accuracy or integrity of any part of the work are appropriately investigated and resolved. Experiments were performed under a project license (No. 150705) granted by the Laboratory Animal Ethics Committee of the Hong Kong Polytechnic University Shenzhen Research Institute, in compliance with the Guangdong Province Experimental Management Regulations for the care and use of animals.

Open Access Statement: This is an Open Access article distributed in accordance with the Creative Commons Attribution-NonCommercial-NoDerivs 4.0 International License (CC BY-NC-ND 4.0), which permits the noncommercial replication and distribution of the article with the strict proviso that no changes or edits are made and the original work is properly cited (including links to both the formal publication through the relevant DOI and the license). See: https://creativecommons.org/licenses/by-nc-nd/4.0/.

\section{References}

1. Saggar JK, Yu M, Tan Q, et al. The tumor microenvironment and strategies to improve drug distribution. Front Oncol 2013;3:154.

2. Nordsmark M, Bentzen SM, Rudat V, et al. Prognostic value of tumor oxygenation in 397 head and neck tumors after primary radiation therapy. An international multicenter study. Radiother Oncol 2005;77:18-24. 
3. Jubb AM, Buffa FM, Harris AL. Assessment of tumour hypoxia for prediction of response to therapy and cancer prognosis. J Cell Mol Med 2010;14:18-29.

4. Campillo N, Falcones B, Otero J, et al. Differential oxygenation in tumor microenvironment modulates macrophage and cancer cell crosstalk: Novel experimental setting and proof of concept. Front Oncol 2019;9:43.

5. Kim H, Lin Q, Yun Z. The hypoxic tumor microenvironment in vivo selects tumor cells with increased survival against genotoxic stresses. Cancer Lett 2018;431:142-9.

6. Soleymani Abyaneh H, Soleimani A, et al. Modulation of hypoxia-induced chemoresistance to polymeric micellar cisplatin: the effect of ligand modification of micellar carrier versus inhibition of the mediators of drug resistance. Pharmaceutics 2018;10:196.

7. Yang F, Zhang H, Mei Y, et al. Reciprocal regulation of HIF-1 $\alpha$ and LincRNA-p21 modulates the Warburg effect. Mol Cell 2014;53:88-100.

8. Xiao Y, Peng H, Hong C, et al. PDGF promotes the warburg effect in pulmonary arterial smooth muscle cells via activation of the PI3K/AKT/mTOR/HIF-1 $\alpha$ signaling pathway. Cell Physiol Biochem 2017;42:1603-13.

9. Samanta D, Park Y, Ni X, et al. Chemotherapy induces enrichment of CD47+/CD73+/PDL1+ immune evasive triple-negative breast cancer cells. Proc Natl Acad Sci U S A 2018;115:E1239-48.

10. Rohwer N, Lobitz S, Daskalow K, et al. HIF-1 $\alpha$ determines the metastatic potential of gastric cancer cells. Br J Cancer 2009; 100:772-81.

11. Oladipupo S, Hu S, Kovalski J, et al. VEGF is essential for hypoxia-inducible factor-mediated neovascularization but dispensable for endothelial sprouting. Proc Natl Acad Sci 2011;108:13264-9.

12. Choi JY, Jang YS, Min SY, et al. Overexpression of MMP-9 and HIF-1 $\alpha$ in breast cancer cells under hypoxic conditions. J Breast Cancer 2011;14:88-95.

13. Shin DH, Dier U, Melendez JA, et al. Regulation of MMP-1 expression in response to hypoxia is dependent on the intracellular redox status of metastatic bladder cancer cells. Biochim Biophys Acta 2015;1852:2593-602.

14. Murdoch C, Giannoudis A, Lewis CE. Mechanisms regulating the recruitment of macrophages into hypoxic areas of tumors and other ischemic tissues. Blood 2004;104:2224-34.

15. Matschurat $S$, Knies UE, Person V, et al. Regulation of EMAP II by hypoxia. Am J Pathol 2003;162:93-103.

16. Casazza A, Laoui D, Wenes M, et al. Impeding macrophage entry into hypoxic tumor areas by Sema3A/ Nrp1 signaling blockade inhibits angiogenesis and restores antitumor immunity. Cancer Cell 2013;24:695-709.

17. Noman MZ, Desantis G, Janji B, et al. PD-L1 is a novel direct target of HIF-1 $\alpha$, and its blockade under hypoxia enhanced: MDSC-mediated T cell activation. J Exp Med 2014;211:781-90.

18. Jayaprakash P, Ai M, Liu A, et al. Targeted hypoxia reduction restores $\mathrm{T}$ cell infiltration and sensitizes prostate cancer to immunotherapy. J Clin Invest 2018;128:5137-49.

19. Noman MZ, Hasmim M, Lequeux A, et al. Improving cancer immunotherapy by targeting the hypoxic tumor microenvironment: new opportunities and challenges. Cells 2019;8:1083.

20. Brooks JM, Menezes AN, Ibrahim M, et al. Development and validation of a combined hypoxia and immune prognostic classifier for head and neck cancer. Clin Cancer Res 2019;25:5315-28.

21. Barsoum IB, Smallwood CA, Siemens DR, et al. A mechanism of hypoxia-mediated escape from adaptive immunity in cancer cells. Cancer Res 2014;74:665-74.

22. Mastelic-Gavillet B, Navarro Rodrigo B, Décombaz $\mathrm{L}$, et al. Adenosine mediates functional and metabolic suppression of peripheral and tumor-infiltrating CD8+ T cells. J Immunother Cancer 2019;7:257.

23. Young A, Ngiow SF, Gao Y, et al. A2AR adenosine signaling suppresses natural killer cell maturation in the tumor microenvironment. Cancer Res 2018;78:1003-16.

24. Gogna R, Madan E, Kuppusamy P, et al. Re-oxygenation causes hypoxic tumor regression through restoration of p53 wild-type conformation and post-translational modifications. Cell Death Dis 2012;3:e286-7.

25. Selvendiran K, Kuppusamy ML, Ahmed S, et al. Oxygenation inhibits ovarian tumor growth by downregulating STAT3 and cyclin-D1 expressions. Cancer Biol Ther 2010;10:386-90.

26. Mast JM, Kuppusamy P. Hyperoxygenation as a therapeutic supplement for treatment of triple negative breast cancer. Front Oncol 2018;8:527.

27. Yu M, Dai M, Liu Q, et al. Oxygen carriers and cancer chemo- and radiotherapy sensitization: Bench to bedside and back. Cancer Treat Rev 2007;33:757-61.

28. Lee NP, Chan KT, Choi MY, et al. Oxygen carrier YQ23 can enhance the chemotherapeutic drug responses of chemoresistant esophageal tumor xenografts. Cancer Chemother Pharmacol 2015;76:1199-207.

29. Qi X, Wong BL, Lau SH, et al. A hemoglobin-based oxygen carrier sensitized Cisplatin based chemotherapy in 
hepatocellular carcinoma. Oncotarget 2017;8:85311-25.

30. Li CX, Wong BL, Ling CC, et al. A novel oxygen carrier "YQ23" suppresses the liver tumor metastasis by decreasing circulating endothelial progenitor cells and regulatory T cells. BMC Cancer 2014;14:293.

31. Gao ZG, Tian L, Hu J, et al. Prevention of metastasis in a 4T1 murine breast cancer model by doxorubicin carried by folate conjugated $\mathrm{pH}$ sensitive polymeric micelles. J Control Release 2011;152:84-9.

32. Nair AB, Jacob S. A simple practice guide for dose conversion between animals and human. J Basic Clin Pharm 2016;7:27-31.

33. Yao Y, Chu Y, Xu B, et al. Risk factors for distant metastasis of patients with primary triple-negative breast cancer. Biosci Rep 2019;39:BSR20190288.

34. Wang Z, Wang $\mathrm{H}$, Sun $\mathrm{X}$, et al. A risk stratification model for predicting overall survival and surgical benefit in triple-negative breast cancer patients with de novo distant metastasis. Front Oncol 2020;10:14.

35. Urru SAM, Gallus S, Bosetti C, et al. Clinical and pathological factors influencing survival in a large cohort of triple-negative breast cancer patients. BMC Cancer 2018;18:56.

36. Yin L, Duan JJ, Bian XW, et al. Triple-negative breast cancer molecular subtyping and treatment progress. Breast Cancer Res 2020;22:61.

37. Kalimutho M, Parsons K, Mittal D, et al. Targeted therapies for triple-negative breast cancer: combating a stubborn disease. Trends Pharmacol Sci 2015;36:822-46.

38. Schito L, Rey S, Tafani M, et al. Hypoxia-inducible factor 1-dependent expression of platelet-derived growth factor B promotes lymphatic metastasis of hypoxic breast cancer cells. Proc Natl Acad Sci U S A 2012;109:E2707-16.

39. Gao JL, Shui YM, Jiang W, et al. Hypoxia pathway and hypoxia-mediated extensive extramedullary hematopoiesis are involved in ursolic acid's anti-metastatic effect in 4T1 tumor bearing mice. Oncotarget 2016;7:71802-16.

40. Zhang H, Lu H, Xiang L, et al. HIF-1 regulates CD47 expression in breast cancer cells to promote evasion of phagocytosis and maintenance of cancer stem cells. Proc Natl Acad Sci 2015;112:E6215-23.

41. Angus L, Smid M, Wilting SM, et al. The genomic landscape of metastatic breast cancer highlights changes in mutation and signature frequencies. Nat Genet 2019;51:1450-8.

42. Yang S, Zhang JJ, Huang XY. Mouse models for tumor metastasis. In: Zheng Y. editor. Rational drug design. Totowa: Humana Press, 2012:221-8.
43. Logsdon DP, Grimard M, Luo M, et al. Regulation of HIF1a under hypoxia by APE1/Ref-1 impacts CA9 expression: Dual targeting in patient-derived 3D pancreatic cancer models. Mol Cancer Ther 2016;15:2722-32.

44. Olive PL, Aquino-Parsons C, MacPhail SH, et al. Carbonic anhydrase 9 as an endogenous marker for hypoxic cells in cervical cancer. Cancer Res 2001;61:8924-9.

45. Schlüter A, Weller P, Kanaan O, et al. CD31 and VEGF are prognostic biomarkers in early-stage, but not in latestage, laryngeal squamous cell carcinoma. BMC Cancer 2018;18:272.

46. Jeon M, Shin Y, Jung J, et al. HIF1A overexpression using cell-penetrating DNA-binding protein induces angiogenesis in vitro and in vivo. Mol Cell Biochem 2018;437:99-107.

47. Musumeci G, Castorina A, Magro G, et al. Enhanced expression of CD31/platelet endothelial cell adhesion molecule 1 (PECAM1) correlates with hypoxia inducible factor-1 alpha (HIF-1 $\alpha)$ in human glioblastoma multiforme. Exp Cell Res 2015;339:407-16.

48. Hay CM, Sult E, Huang Q, et al. Targeting CD73 in the tumor microenvironment with MEDI9447. Oncoimmunology 2016;5:e1208875.

49. Loi S, Pommey S, Haibe-Kains B, et al. CD73 promotes anthracycline resistance and poor prognosis in triple negative breast cancer. Proc Natl Acad Sci U S A 2013;110:11091-6.

50. Buisseret L, Pommey S, Allard B, et al. Clinical significance of CD73 in triple-negative breast cancer: Multiplex analysis of a phase III clinical trial. Ann Oncol 2018;29:1056-62.

51. Allard D, Chrobak P, Allard B, et al. Targeting the CD73adenosine axis in immuno-oncology. Immunology Letters 2019;205:31-9.

52. Lee J, Ahn E, Kissick HT, et al. Reinvigorating exhausted T cells by blockade of the PD-1 pathway. For Immunopathol Dis Therap 2015;6:7-17.

53. Ohta A, Kini R, Ohta A, et al. The development and immunosuppressive functions of CD4+ CD25+ FoxP3+ regulatory $\mathrm{T}$ cells are under influence of the adenosineA2A adenosine receptor pathway. Front Immunol 2012;3:190.

54. Ma SR, Deng WW, Liu JF, et al. Blockade of adenosine $\mathrm{A} 2 \mathrm{~A}$ receptor enhances $\mathrm{CD} 8+\mathrm{T}$ cells response and decreases regulatory $\mathrm{T}$ cells in head and neck squamous cell carcinoma. Mol Cancer 2017;16:99.

55. Turcotte M, Allard D, Mittal D, et al. CD73 promotes 
resistance to HER2/ErbB2 antibody therapy. Cancer Res 2017;77:5652-63.

56. Moen I, Stuhr LEB. Hyperbaric oxygen therapy and cancer - a review. Target Oncol 2012;7:233-42.

57. Yttersian Sletta K, Tveitarås MK, Lu N, et al. Oxygendependent regulation of tumor growth and metastasis in human breast cancer xenografts. PLoS One 2017;12:e0183254.

58. Raa A, Stansberg C, Steen VM, et al. Hyperoxia retards growth and induces apoptosis and loss of glands and blood vessels in DMBA-induced rat mammary tumors. BMC Cancer 2007;7:23.

59. Moen I, Tronstad KJ, Kolmannskog O, et al. Hyperoxia increases the uptake of 5-fluorouracil in mammary tumors independently of changes in interstitial fluid pressure and tumor stroma. BMC Cancer 2009;9:446.

60. Suh JH, Stea B, Nabid A, et al. Phase III study of efaproxiral as an adjunct to whole-brain radiation therapy

Cite this article as: Chow E, Lau JSH, Wai T, Lam IPY. The anti-tumoral effects of the oxygen carrier YQ23 in a triplenegative breast cancer syngeneic model. Transl Cancer Res 2021;10(2):656-668. doi: 10.21037/tcr-20-2768 for brain metastases. J Clin Oncol 2006;24:106-14.

61. Gainer JL, Sheehan JP, Larner JM, et al. Trans sodium crocetinate with temozolomide and radiation therapy for glioblastoma multiforme. J Neurosurg 2017;126:460-6.

62. Johnson JLH, Leos RA, Baker AF, et al. Radiosensitization of Hs-766T pancreatic tumor xenografts in mice dosed with dodecafluoropentane nano-emulsion-preliminary findings. J Biomed Nanotechnol 2015;11:274-81.

63. Bowman CE, da Silva RG, Pham A, et al. An exceptionally potent inhibitor of human CD73. Biochemistry 2019;58:3331-4.

64. Willingham SB, Ho PY, Hotson A, et al. A2AR antagonism with CPI-444 induces antitumor responses and augments efficacy to anti-PD-(L)1 and anti-CTLA-4 in preclinical models. Cancer Immunol Res 2018;6:1136-49.

65. Young A, Ngiow SF, Barkauskas DS, et al. Co-inhibition of CD73 and A2AR adenosine signaling improves anti-tumor immune responses. Cancer Cell 2016;30:391-403. 